\title{
LA PARTICIPACIÓN POLÍTICA DE LA MUJER EN LA PLANIFICACIÓN DE LA CIUDAD
}

\section{THE POLITICAL PARTICIPATION OF WOMEN IN CITY PLANNING}

\author{
Yeinis Cantillo Peralta ${ }^{(1)}$ \\ Universidad del Rosario, Bogotá, Colombia \\ Universidad de Buenos Aires, Argentina
}

\begin{abstract}
Resumen: La planificación de las ciudades ha sido el principal instrumento de organización para las ciudades, mas, sin embargo, no se incluyen en él las repercusiones y problemáticas que estas transformaciones urbanas provocan en el género femenino. Por ello, se considera fundamental analizar y desnaturalizar las construcciones discursivas de dominación masculina que afectan a las mujeres en el empoderamiento a cargos públicos relacionados con la planificación estratégica de las ciudades y en la materialización de instrumentos jurídicos que tengan en cuenta la perspectiva de género.
\end{abstract}

Palabras claves: Género, urbanismo, democracia participativa, derecho emergente, política.

Abstract: The city's planning has been the main instrument of organization for the cities, but the repercussions and problems that these urban changes have had for the feminine group are not taken into acount. Therefore, it's important to analyze and desnaturalize the patriarchal discourse that affect women in the empowerment of public office related to the city strategic planning, and in the establishment of legal instruments that takes into account the gender perspective.

Key words: gender, urbanism, participation democracy, emerging human rights, politics.

(1) Abogada de la Universidad del Rosario, Bogotá. Colombia. Cursando la Maestría Planificación Urbana y Regional en la Universidad de Buenos Aires, Argentina. 



\section{Introducción}

La incorporación de la perspectiva de género en la producción del hábitat urbano en los países a nivel mundial no ha sido homogénea. En los países anglosajones la discusión de género lleva más de 30 años mientras que en nuestros países latinoamericanos ha sido no solo tardía sino parcial, aun así, encontramos una similitud en ambos y es que en este momento y en todo el mundo, existen mujeres que cada vez más ocupan cargos importantes, sin embargo, las decisiones en cuanto a la planificación urbana es principalmente de los hombres.

De esta manera las mujeres son doblemente excluidas como ciudadanas y portadoras de un derecho emergente como el derecho a la ciudad, en tanto que son invisibilizadas de las necesidades que ellas requieren y de los demás colectivos marginalizados, como también en la tarea de planificar.

Si bien es cierto que, las mujeres han sido participes de los procesos de construcción de sus ciudades, aportando a mejorar las condiciones de hábitat, particularmente los movimientos sociales quienes han demandado al Estado, vivienda, servicios, transporte, en la construcción y mejoramiento, no son ellas las que al final del día toman la decisión presente y futura en las políticas públicas de la ciudad. Por lo que se presenta un primer desafío, el de vincular la planificación no como un proceso técnico sino también un proceso político. Así mismo, reconocemos que la Comunidad Internacional ha realizado numerosas Recomendaciones sobre temática de género y hábitat urbano, pero, todavía falta el apoyo sistemático y abarcativo de un derecho real y participativo de las mujeres en la ciudad en cuanto a las planificadoras urbanas, y en general a las mujeres relacionados en estos cargos.

El propósito de este artículo busca exponer, así como sensibilizar, la incorporación de las representaciones de las mujeres en el Derecho y en el poder político por la importancia en el desarrollo y en la construcción de las ciudades sostenibles.

El artículo se compone de una reflexión corta de la mujer en la historia según Lipovetsky (2007), para ahondar en las dicotomías geográficas como lo públicoprivado, movilidad-inmovilidad, las representaciones espaciales de lo femenino y lo masculino como construcciones ideológicas que afectan directamente el ordenamiento urbano. Una segunda reflexión muestra la representación política de las mujeres en la actualidad para posteriormente hacer unas breves reflexiones acerca del Derecho a la ciudad y finalmente un acápite final de conclusiones.

\section{La Mujer moderna y sus tres paradigmas según Lipovetsky (2007)}

El filósofo y sociólogo francés Gilles Lipovetsky considerado "el heredero de Tocqueville y Louis Dumont" (Luc Ferry) o la estrella de los analistas de la 
contemporaneidad (Vicent Verdù). Expone las tesis de su último libro: "La Tercera Mujer: permanencia y revolución de lo feminismo". Este autor hace un recorrido de la historia de la mujer en la humanidad dividiéndolo en tres paradigmas para explicar a la mujer actual y su rol político.

Durante el periodo más largo de la historia de la humanidad la mujer fue considerada como un mal necesario, un ser inferior, sistemáticamente despreciada por la sociedad. Desde el momento en que los trabajos se dividieron en roles definidos para hombres y para mujeres, esta distribución no fue equitativa ni simétrica, sino que se dotó al hombre de valores superiores y positivos resaltándolos en sus cargos políticos y sus funciones en la guerra; mientras que las labores femeninas se consideraron inferiores y negativas, señalándolas solo por su función procreadora y direccionándolas a la maternidad. Sin embargo, es el hombre el único dador de vida y la mujer la cuidadora.

En este primer paradigma, la mujer es un reflejo de la fuerza del mal que agrede el orden social. Se le describe como seres engañosos, inconstantes, envidiosos (Daros, 2014) que permanecen en la sombra y en el olvido. Su participación en la ciudad y en las calles es deshonrosa incentiva al hombre a "pecar" al deleite de las pasiones por lo que es necesario limitarse a convivir en una vida privada, para dedicarse a los futuros hombres que tendrán una gloria inmortal y honores públicos.

Un cambio cultural e histórico muy importante ocurre después de la segundad edad media a partir del Código del amor cortesano que rendía culto a la dama amada y exacerbada a sus perfecciones morales y estéticas. Según el autor a partir del siglo XII, el Renacimiento lleva a su apogeo este paradigma donde Dante idealiza a Beatriz, Don Quijote dedica sus hazañas a su hermosa dama Dulcinea. En los siglos posteriores, la llustración continua con la exaltación, alabando los méritos de las mujeres y sus aportes al mejoramiento del buen vivir. La mujer es acá idealizada, alabada y sacralizada (Daros, 2014) aunque esta situación no cambiase la realidad de la mujer que siguió confinada a su espacio privado, sin independencia económica y sin desempeñar papel alguno en la política.

Ya en los siglos XVIII y XIX es la esposa, madre, y educadora a de los niños a la que ponen en pedestal filósofos, ideológicos y poetas. De acuerdo al análisis de Lipovetsky esa es la segunda mujer no reconocida como sujeto igualitario y autónomo pero cuyos roles son reconocidos socialmente.

Se da entonces un tercer paradigma: la mujer indeterminada o pos mujer. Desde mediados del siglo XX, la mujer ya no es definida por la mirada del hombre. Ahora, la mujer se lanza a la posibilidad de ser lo que ella desea ser. Se abre las puertas a la mujer que adquiere derechos tales como; el sufragio, el divorcio, a la 
libertad sexual y al control sobre la procreación. Así, la mujer tiene el poder de inventarse a sí misma, aunque esto no supone la desaparición de desigualdades entre los sexos, tampoco al reconocimiento de la igualdad de derechos, ni mucho menos a un estado de intercambio de roles y lugares.

A pesar del apogeo de la mujer en su reivindicación de derechos, la feminización de las carreras y el aumento de empleo para y hacia las mujeres, el poder económico y político permanece mayoritariamente en manos masculinas. Si continuamos la lógica de que lo privado está asociado a la mujer y lo público al hombre, esto nos conlleva indiscutiblemente a ver las consecuencias en la ciudad. Una ciudad definida por hombres, donde los valores públicos están priorizados por ellos, donde el territorio se define conforme a las necesidades del hombre y sin tener en cuenta las prioridades de la mujer del siglo XXI, de aquella que percibe el espacio como medio de transición entre lo público y lo privado, que requiere de él para mantener su empleo acortando la distancias entre su casa y la oficina, acercando el jardín de niños con su empleo o priorizando al peatón con amplias veredas donde se pueda caminar con el coche del bebé.

\section{La planificación urbana con perspectiva de género}

El espacio construido a través de las prácticas sociales, por la lucha y apropiación de una porción del territorio se da en un espacio determinado, en un momento determinado e histórico. Por eso el modelo espacial actual, responde todavía a la economía capitalista, basada en una división tradicional de roles de sexos y en una estructura social tradicional. En donde -como hemos mencionadolo privado es de la mujer mientras que lo público es del hombre lugar de trabajo y desarrollo económico. Por tal motivo prevalece todavía en la mayoría de las ciudades latinoamericanas la atención al vehículo privado, la segregación de usos del espacio, los desarrollos de vivienda dispersa y sin cohesión social, la perdida, en definitiva, "de los valores tradicionales de la ciudad como espacio colectivo rico y diverso en posibilidades de intercambio social" (Garcia Nart, 1996).

En un ensayo clásico de 1980 llamado ¿Cómo sería una ciudad no sexista? La urbanista estadounidense Dolores Hayden, pone a consideración los problemas logísticos que enfrentan las mujeres empleadas en Estados Unidos, problemas que no se pueden considerarse del ámbito privado y no sucumben a las soluciones del mercado (Hayden, 1981) es decir, los problemas distancia-tiempo, las guarderías de los niños y la renta de la vivienda no son cuestiones privadas o de financiamiento son problemas de corte urbano y de gestión de la ciudad ${ }^{(2)}$.

(2) "If architects and urban designers were to recognize all employed women and their families as a constituency for new approaches to planning and design and were to reject all previous assumptions about 'woman's place' in the home, what could we do? Is it possible to build non-sexist neighborhoods and design non-sexist cities? What would they be like?" (Hayden, 1981) 
Si los arquitectos y los diseñadores urbanos reconocieran a todas las mujeres empleadas y sus familias como constituyentes para los nuevos enfoques de planificación y diseño y rechazaran todas las suposiciones anteriores sobre "el lugar de la mujer" en el hogar, ¿Qué podríamos hacer? ¿es posible construir vecindarios no sexistas y diseñar ciudades no sexistas? ¿Cómo serían? (Hayden, 1981)

Por ello, la aportación con enfoque de género en el planeamiento y políticas urbanas territoriales, tienen una importancia fundamental en la construcción de las ciudades en un doble sentido. En primer lugar, reconociendo que el espacio históricamente no es neutro (Muxi, 2011) por lo que requiere de la reivindicación general del Derecho a la ciudad como espacio de convivencia que permita y garantice unas condiciones adecuadas a la calidad de vida para todos incluyendo vivienda, equipamientos, servicios, entorno y medio ambiente saludable etc. (García, 1996), dejando a un lado la visión androcéntrica que se tiene de la ciudad, donde arquitectos como Le Corbusier reconocieron que era indiscutible la necesidad de trabajar la ciudad a escala humana, pero escala medida sobre 6 pies de altura o 1.82 metros promedio superior a las mujeres (Guardian, 2014).

En segundo lugar, porque si el fin de la planificación urbana es garantizar una adecuada calidad de vida, propiciar a una "justicia social" o por lo menos intentar integrar las interrelaciones, espaciales, socio-culturas, económicas y ambientales de las ciudades en soluciones a problemas urbanos complejos a partir de políticas urbanas, programas territoriales e instrumentos de planificación, es evidente que para ellos es preciso analizar y conocer los problemas, necesidades y demandas concretas de cada grupo social sin las presiones patriarcales (Muxi, 2011).

La antropóloga urbana Caroline Moser, "hace la distinción entre las necesidades prácticas de género, como las sillas altas para bebes o baños y las estratégicas como la representación política, o instalaciones recreativas solo para mujeres" (Guardian, 2014)

A este primer punto, damos como ejemplo la diferencia sustancial en el uso de los medios de transporte en ambos sexos. Por ejemplo, el estudio Gender, Travel and Job Access; evidence from Buenos Aires del Banco Mundial declara que en un $25 \%$ de los viajes que realizan las mujeres en Buenos Aires son a pie $y$, en la mayoría de las ciudades de la región, las mujeres son las principales usuarias del transporte público. En cambio, los hombres son los principales usuarios de los automóviles (Bank, 2014). Las mujeres se ven obligadas a movilizarse a pie por el costo de comprar un automóvil o tomar taxis todos los días, los sistemas no responden a sus necesidades de movilidad y por último por que el transporte público no suele llegar a donde las mujeres quieren ir (guarderías, centros médicos o áreas donde ellas trabajan. (Monjesilva, 2016) 
El desajuste entre la organización territorial y las necesidades de accesibilidad, y movilidad de las mujeres, incrementa las dificultades para compatibilizar los tiempos domésticos familiares y laborales remunerados, impactando en su calidad de vida y condicionando las decisiones personales (Rainer \& Marisol, 2011). Asunto no menor este, si se tiene en cuenta que en América Latina en el 2005, un 13,1\% correspondía a un hogar de tipo monoparental, de los cuales el 86,8\% estaba encabezado por mujeres (Cepal, 2014). Desde un punto de vista socioeconómico y cultural, el incremento de hogares comandados por mujeres es importante teniendo en cuenta que América Latina es una de las regiones más desiguales del mundo -en términos económicos, étnicos y sociales(Cepal, 2014) y que este rasgo tiene gran incidencia en la manera del uso y goce de la ciudad.

Así mismo el Banco Interamericano de Desarrollo considera que las mujeres líderes que atienden asuntos urbanos tienden a tener un estilo de negociación y liderazgo más inclusivo y menos jerárquico que sus homólogos masculinos; segundo, porque las mujeres son más conscientes de "planificar y diseñar espacios públicos más seguros para todos los grupos sociales requiere no solo elementos de hardware (iluminación, paisajismo, mobiliario urbano, señalización, entre otros, sino también elementos de software o políticas públicas que tomen en consideración las necesidades y preocupaciones de las mujeres)" (BID, 2016); y tercero porque las mujeres están más ligadas a avances en campos tales como la educación, la infraestructura y la salud a nivel local.

La perspectiva de género entonces, nos permite incorporar factores claves a los análisis y políticas urbanísticas-territoriales, todos ellos con el reto fundamental que se plantea para el futuro de conseguir unas ciudades más sostenibles, considerando como tales las ciudades que tengan una visión integral de los problemas y satisfacer las necesidades que incluyen vivienda, equipamientos, transportes etc., como parte de un todo para garantizar derechos fundamentales.

\section{Las mujeres en las tomas de decisión}

La reivindicación de derechos de las mujeres en la ciudad y la conciencia de la perspectiva de género, no residen en tomar conciencia del lenguaje y en el conocimiento del derecho porque "el principio de visión dominante no es una simple representación mental, un fantasma, una ideología, sino un sistema de estructuras establemente inscriptas en las cosas y en los cuerpos" (Bourdieu, 2010, pág. 57). Así pues, es necesaria la presencia de las mujeres en los procesos de toma de decisiones que pueden resultar clave para la defensa de la equidad de género, movilidad urbana e integración de las necesidades de ellas en los planos nacionales de ordenamiento territorial, en los planes estratégicos de la ciudad y hasta en la norma de uso del suelo. 
No se trata solo de una ideología sino de "poder estar" presentes en las decisiones que afectan la vida cotidiana de las mujeres. La tercera mujer de Lipovetsky (2007) asume cargos públicos por causa que conduzcan a un "sentido progreso para los otros, que expresen un ideal común [en vez de] sacrificarse por funciones económicas marcadas sobre todo por el gusto del poder por el poder." (Lipovetsky, 2007 , pág. 252)

Las mujeres políticas "son más pragmáticas y menos arribistas que los hombres, se muestran menos fascinadas que ellos por el juego del poder, no les preocupa tanto alcanzar puestos como imponer sus ideas y realizar avances concretos" (Lipovetsky, 2007 , pág. 260). El poder para la mujer según el filósofo francés, se contempla como un medio más que como un fin en sí mismo.

Para ello, existe un marco jurídico en donde la comunidad internacional ha subrayado la importancia de darle prioridad a la problemática de género y de reconocer la existencia de unos derechos y obligaciones para los Estados en la planificación de políticas y estrategias de desarrollo. Las ultimas conferencias como; la Conferencia de las Naciones Unidas sobre Medio Ambiente y Desarrollo celebrada en Rio de Janeiro en 1992 abordó explícitamente aspectos relativos al género en la Agenda 21, Plataforma de la Cumbre de la Tierra para Futuras Acciones. También en la Conferencia Mundial sobre Derechos Humanos en Viena 1993, se lograron importantes adelantos en el reconocimiento de los derechos de la mujer, reafirmando que los derechos de las mujeres y niñas son parte integral, inalienable e indivisible de los derechos humanos universales. Este principio fue asimismo uno de los objetivos básicos de la Conferencia Internacional sobre Población y Desarrollo, celebrada en el Cairo en 1994.

En la cumbre Mundial sobre Desarrollo Social de Copenhague en 1995, la problemática relativa al género fue el eje de todas las estrategias para el desarrollo social, económico y la conservación del medio ambiente. Por último, la Cuarta Conferencia Mundial sobre la Mujer celebrada en 1995 en Beijing, donde se adoptó la Declaración y la Plataforma de Acción de Beijing en materia de eliminación de todos los obstáculos para lograr la igualdad entre hombres y mujeres y asegurar la participación activa de todas las mujeres en todas las esferas de la vida.

Estos esfuerzos internacionales y nacionales han dado como respuesta la Convención sobre la Eliminación de Todas las Formas de Discriminación Contra la Mujer 1997 (CEDAW, por sus siglas en ingles), cuando establece que los Estados partes deben de garantizar que las mujeres participen en "la formulación de las políticas gubernamentales y en la ejecución de éstas, y ocupar cargos públicos y ejercer todas las funciones públicas en todos los planos gubernamentales" (CEDAW, 1997) reconociendo en la Recomendación General 23 que: 
"Ni siquiera las democracias históricamente estables han podido integrar (...) las opiniones y los intereses de la mitad femenina de la población. No puede llamarse democrática una sociedad en la que la mujer este excluida de la vida pública y del proceso de adopción de decisiones. El concepto de democracia tendrá significación real (...) cuando hombres y mujeres compartan la adopción de decisiones políticas y cuando los intereses de ambos se tengan en cuenta por igual". (CEDAW, 1997)

Actualmente, la presencia política de las mujeres latinoamericanas ha alcanzado los más altos indicadores con senadoras, diputadas, ministras, alcaldesas, concejales. El mapa de la UIP-ONU sobre las Mujeres en la Política, contiene la clasificación mundial relativa a las mujeres en el poder ejecutivo y en la rama legislativa de los gobiernos al 1 de enero de 2019 , en donde se muestra que la proporción de ministras mujeres se encuentra en un nivel alto sin precedentes (ONU M. , 2019) con el 20,7\%, 2,4 puntos porcentuales más con respecto a 2017. También se muestra que se diversificaron los tipos de cartera de los cuales son titulares las mujeres ministras.

El siguiente cuadro muestra todos los países de Latinoamérica con su rango a nivel mundial y el porcentaje de mujeres ministras.

Tabla 1. Mujeres latinoamericanas con cargo ministeriales

\begin{tabular}{|c|c|c|c|c|}
\hline $\begin{array}{c}\text { Rango a nivel } \\
\text { mundial }\end{array}$ & País & \% mujeres & Mujeres & Total de ministros \\
\hline \multicolumn{5}{|c|}{60 a $69,9 \%$} \\
\hline 2 & Nicaragua & 55,6 & 10 & 18 \\
\hline 5 & Colombia & 52,9 & 9 & 17 \\
\hline 6 & Costa Rica & 51,9 & 14 & 27 \\
\hline \multicolumn{5}{|c|}{40 a $49,9 \%$} \\
\hline 13 & Uruguay & 42,9 & 6 & 14 \\
\hline 16 & México & 42,1 & 8 & 19 \\
\hline \multicolumn{5}{|c|}{30 a $34,9 \%$} \\
\hline 30 & Chile & 34,8 & 8 & 23 \\
\hline 36 & El Salvador & 33,3 & 5 & 25 \\
\hline
\end{tabular}




\begin{tabular}{|c|c|c|c|c|}
\hline & & & 25 a $29,9 \%$ & \\
\hline 43 & Venezuela & 29,4 & 10 & 34 \\
\hline 53 & Haití & 27,8 & 5 & 18 \\
\hline 53 & Perú & 27,8 & 5 & 18 \\
\hline 60 & Panamá & 26,7 & 4 & 15 \\
\hline 64 & Argentina & 25 & 3 & 12 \\
\hline 64 & Cuba & 25 & 7 & 28 \\
\hline \multicolumn{5}{|c|}{20 a $24,9 \%$} \\
\hline 83 & Ecuador & 22,2 & 6 & 27 \\
\hline 90 & Paraguay & 21,4 & 3 & 14 \\
\hline \multicolumn{5}{|c|}{15 a $19,9 \%$} \\
\hline 111 & $\begin{array}{l}\text { República } \\
\text { Dominicana }\end{array}$ & 16,7 & 4 & 24 \\
\hline \multicolumn{5}{|c|}{10 a $14,9 \%$} \\
\hline 123 & $\begin{array}{c}\text { Bolivia } \\
\text { (Estado } \\
\text { Plurinacional) }\end{array}$ & 14,3 & 3 & 21 \\
\hline \multicolumn{5}{|c|}{5 a $9,9 \%$} \\
\hline 149 & Brasil & 9,1 & 2 & 22 \\
\hline 160 & Guatemala & 6,7 & 1 & 15 \\
\hline
\end{tabular}

Fuente: elaboración propia tomado de MAPAS ONU-Mujeres ${ }^{(3)}$

Hemos de resaltar que respecto a mujeres parlamentarias entre los 10 primeros países a nivel mundial se encuentran 5 países latinoamericanos en el siguiente orden: Cuba (2), Bolivia (Estado Plurinacional De) (3), México (4), Costa Rica (8) y Nicaragua (9) sin embargo, no ahondaremos en esto por cuanto consideramos que el cargo de senadora o parlamentaria asume un abanico de temas que no se sujetan exclusivamente a temas urbanos.

Con respecto a las 1412 carteras en 188 países de las que son titulares las ministras y en cuanto a asuntos relacionados a temas de Planificación Urbana y Regional, encontramos que a nivel mundial se encuentran 53 en obras públicas/ planificación del territorio, 25 en vivienda/asuntos urbanos y 19 en transporte, que solo representa el $7 \%$.

(3) Infografía. Disponible en:https://www.ipu.org/resources/publications/infographics/2019-03/ women-in-politics-2019 
Ilustración 1. Carteras de las que son titulares ministras a nivel mundial

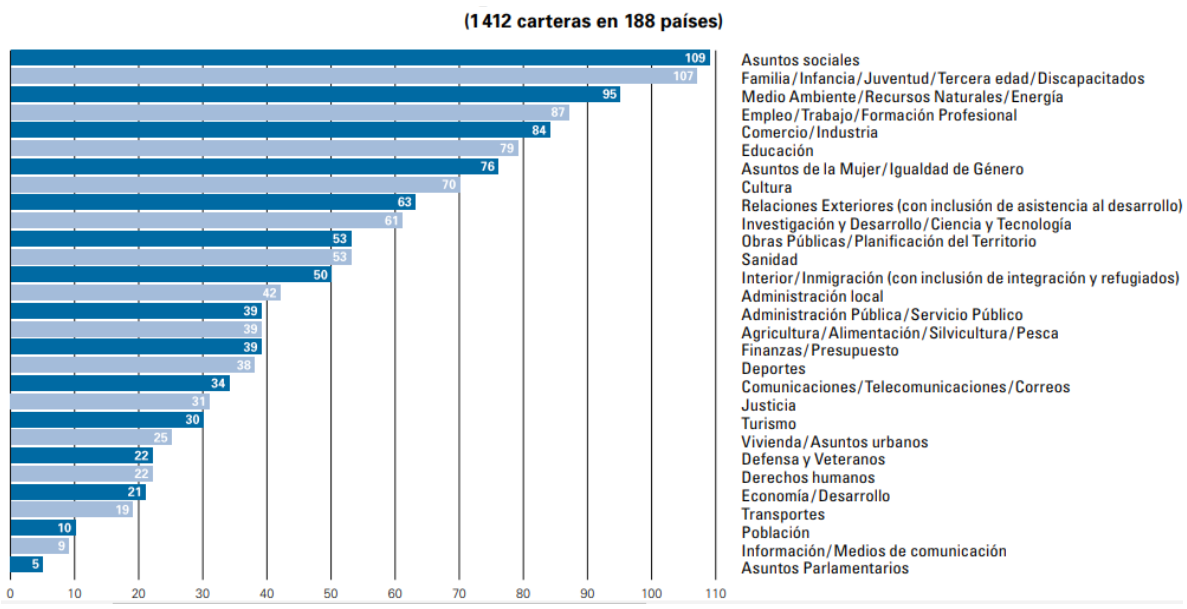

Fuente: Mapas ONU-Mujeres ${ }^{(\mathbf{4})}$

Por lo que continúan ocupándose cargos tradicionales como la Educación, la salud, asuntos de género, cultura y asuntos sociales, sin desconocer que en este momento más mujeres desempeñan cargos que tradicionalmente eran desempeñados por hombres con respecto al 2017 por ejemplo, existe un $30 \%$ más de mujeres ministras que desempeñan el Ministerio de Defensa, un 52\% más de mujeres en el Finanzas y un 13,6\% más de mujeres en el de Relaciones Exteriores (ONU M. , 2019).

Es innegable que la participación de las mujeres además de ser minoritarias en la política se le añade que se les restringe a sectores considerados femeninos "raras son las ministras que ejercen las funciones de regalías" (Lipovetsky, 2007, pág. 235), frente a esta limitación de la representación política una de sus explicaciones es por el regreso a la mujer protegida considerada limitada para ejercer dichos asuntos complejos. Adriana Salvatierra de 29 años, Presidenta de la Cámara de senadoras de Bolivia, la mujer más joven en ocupar este cargo en su país Bolivia y en América Latina explica que (ONU, 2019): "todavía es difícil para las mujeres participar en la política hoy, especialmente si eres una mujer joven, debido a todos los perjuicios que enfrentamos (...) Hay una sobreprotección de las mujeres, que en última instancia nos subestima y nos subvalora".

Así pues, podría afirmarse que la incursión femenina en la esfera pública continúa siendo principalmente mediante la movilización comunitaria, trabajando por el desarrollo y luchando por los derechos de los grupos más desfavorecidos

(4) IBID. 
(Tello \& Silvia, 2009). Lo que ha contribuido en fortalecer las relaciones entre el Estado y la sociedad civil, en las cuales las mujeres han ejercido un gran poder de influencia en las políticas públicas, en la incorporación de las problemáticas identificadas en las ciudades a las agendas de los gobiernos, lo que ha repercutido en una gradual conciencia de género y de circunstancias socio-económicas particulares. La lucha de Jane Jacobs, por proteger "el village" en el Bajo Manhattan, es un claro ejemplo de lo que logra el activismo social donde ella buscaba no solo preservar un paisaje urbano sino también evitar la expansión descontrolada de las urbes, la construcción de grandes autovías, la separación de los barrios y el uso del automóvil como principal modo de transporte, hasta condensar su esfuerzo en el libro más reconocido The Death and Life of Great American Cities publicado en 1961.

Esta manera de participar en la política pública, apuntala a dos tipos de activistas. Aquellas que, de todas maneras, colocan su participación en la lucha por una construcción democrática sin llegar a decidir en ella (Ranaboldo \& solano, 2008). Y otras que les ha servido como punto de partida y vehículo para los sistemas políticos formales. Lo anterior quedo demostrado en la investigación realizada por (Tello \& Silvia, 2009) donde el 76,2\% de las alcaldesas y concejalas latinoamericanas manifestó haber participado en organizaciones no gubernamentales o con fines apolíticos con anterioridad a sus carreras.

Así mismo, la descentralización y la desconcentración del poder se han señalado como el mecanismo idóneo en las relaciones ente Estado y sociedad por lo que se abren nuevas oportunidades para la participación social contribuyendo a que disminuya el desafío de la democracia real en promover la participación de las mujeres, en reconocerlas como sujetos de derechos y puedan ser incorporadas en las instituciones donde se toman las decisiones (CEPAL, 2007).

En este sentido, son los municipios los principales espacios idóneos de organización gubernamental capaz de captar las problemáticas e idear métodos apropiados para responder a las especificidades de cada segmento social a ser atendido (Tello \& Silvia, 2009). Es el municipio quien identifica y soluciona los problemas menos complejos y con mayor alcance que los que se dan en el ámbito nacional.

De manera que la posibilidad de las mujeres de trabajar en las administraciones públicas hace que tengan una incidencia efectiva en las políticas públicas, en la inclusión, deliberación y corresponsabilidad diferenciada, en llegar a pensar la vivienda en relación a la calidad del tejido urbano para construir mejores ciudades. Así se llega a una gran red comunicacional conformada por una relación ciudad-mujeres-planes urbanos, para que su gestión tenga un efecto multiplicador. 


\section{Trayendo a colación el derecho a la ciudad}

Mucho se ha hablado acerca de los derechos humanos emergentes o los derechos de cuarta generación, entre ellos el Derecho a la ciudad. En este último apartado queremos hacer una breve reflexión acerca del reto que trae el derecho a la ciudad como medio para la participación de las mujeres en la toma de decisiones.

Se entiende como derechos emergentes aquellos derechos que surgen de la evolución de las sociedad, algunos nuevos (derecho al espacio radioeléctrico), otros que ya se encuentran positivizados en el Sistema Internacional de Protección de los Derechos Humanos pero que habían sido olvidados o su contenido se ha ampliado y concretado (Guillén Lanzarote, 2011). Además, otra característica de los Derechos Humanos es su aplicación a colectivos que anteriormente no disfrutaban de un derecho concreto como la participación política de los inmigrantes.

Los Derechos Humanos Emergentes tienen como finalidad la de formar parte de los derechos fundamentales, no obstante este derecho no forma parte del sistema ni a nivel estatal o supraestatal, solamente se encuentran reconocidos en textos políticos o no jurídicamente vinculantes como la Carta Mundial sobre el Derecho a la Ciudad o son reconocidos de forma transversal como en la Constitución brasileña de 1988 y la Constitución colombiana de 1991, que fueron los primeros documentos constitucionales latinoamericanos que otorgaron derechos fundamentales a los derechos urbanos, con la salvaguarda de derecho a la vivienda y al derecho al acceso de agua potable. En efecto, la Corte Colombiana ha reiterado la tutela del derecho a la vivienda a través de la conexidad con otros derechos fundamentales como la vida, la dignidad humana y la salud. En cuanto al derecho al agua lo ha reconocido como un derecho fundamental y un servicio público donde el municipio debe garantizar el acceso efectivo al agua potable, salubre y de calidad ${ }^{(5)}$.

La doctrina ha hecho una distinción entre los derechos a la ciudad y los derechos humanos en la ciudad. Estos últimos son aquellos derechos que se dan en un espacio geográfico determinado mientras que los derechos a la ciudad son acciones de las autoridades locales, "es decir, cuando reivindicamos el derecho a la ciudad estamos revindicando un espacio colectivo donde se respeten los derechos humanos, estamos reivindicando los derechos humanos en la ciudad." (Guillén Lanzarote, 2011)

(5) Según la jurisprudencia actual de la Corte Constitucional (T118/08, T616/10, T1089/12 entre otras) el derecho fundamental al agua puede ser amparado a través de la acción de tutela de manera independiente cuando el acceso de una persona a este recurso, para su uso personal o doméstico, se ve afectado en alguna de las condiciones mínimas establecidas por la Observación General No. 15 del CDESC. 
La conceptualización así entendida nos lleva a una dificultad, y es que, el derecho a la ciudad es ideológico ${ }^{(6)}$ (Guillén Lanzarote, 2011), amplio y abarca a una serie de derechos constitucionales y convencionales que solo pueden ser ejercidos en la ciudad, y que en últimas se direcciona a una falta de claridad dogmática.

Por ello, el derecho a la ciudad complejiza la participación de las mujeres al no ser claro en la manera en que complementa la participación de las mujeres en la toma de decisiones, llegando a un vacío jurídico normativo. Si bien el derecho a la ciudad busca ampliar la calidad de vida de las personas a escala urbana, los mecanismos de protección, promoción y salvaguarda de ellos deben ser claros, para los Estados partes y poder unir la universalidad de derechos que tienen las mujeres en la ciudad, ya sea la capacidad de gestión urbana con perspectiva de género, la reivindicación del espacio público para los colectivos o la participación de las mujeres en la política sectorial.

Por el momento el Derecho a la ciudad, es un paradigma que permite evaluar el grado de la democracia participativa y las condiciones en que se dan los derechos colectivos para "permitir ubicar la condición humana en un valor moral superior". (Guillén Lanzarote, 2011).También es cierto que, la articulación de la ciudad como derecho humano no tiene mucho tiempo de existencia, sin embargo, recalcamos que su codificación o proceso para ser parte del Sistema de Protección de los Derechos Humanos requiere abarcar el Derecho de forma integral, multiescalar y multidimensional.

\section{Conclusión}

La ciudad ha sido desarrollada a partir de la asimetría entre hombres y mujeres, según una visión androcéntrica, en donde el espacio cobra importancia como escenario activo de las relaciones para la reivindicación de los derechos y la herramienta esencial para facilitar, o por el contrario obstruir, las necesidades de cada grupo poblacional.

El derecho a la ciudad tiene una estrecha relación con los derechos a la vivienda, espacio público, movilidad, visibilidad del tejido urbano e identidad de los lugares como símbolo de apropiación.

De este conjunto de análisis se puede apreciar que las mujeres Ministras que ocupan cargos relacionados con la planificación y el desarrollo urbano solo representan el $7 \%$ del total de Ministras en el mundo. Por lo que ¿construir ciudades, o descontruirlas, con nuevas viviendas, calles, oficinas serian o se sentirían

(6) Por eso para Lefebre el urbanismo no podía llegar hacer un sistema porque es una ideología donde existen diferentes actores cada uno con pensamientos distintos sobre la ciudad. "por eso existen diferentes urbanismos: el del racionalismo ilustrado, el de los humanistas, el de los promotores el de los tecnócratas [... ]" (Gonzalez, 1998) 
diferentes si más mujeres estuvieran a cargo de diseñarlas? No lo sabemos, pero lo que si creemos es que en ningún lugar del mundo las mujeres tienen el poder político o el acceso al capital para una planificación urbana de género, que al final siguen siendo solo recomendaciones para un sector político conformado en su mayoría por hombres que decidirán en un papel protector los temas que consideran importantes y los que no.

Si esto suena utópico, el derecho a la ciudad entonces, tiene un largo camino por recorrer al necesitar reconocer que las capacidades no se construyen en abstracto, que es necesario la implementación de directrices para la titularidad de las mujeres y una participación efectiva en el ejercicio pleno de la ciudadanía universal o política, para que las problemáticas analizadas y visualizadas por los movimientos sociales y las mujeres tengan una dirección más productiva para la discusión política entorno al espacio construido, así seguramente, no se pierda de vista resolver problemas tan urgentes como la inasequibilidad y el aumento de vivienda o la distancias entre hogar y lugar de trabajo. En este sentido, la participación de las mujeres será una condición sine qua non de la gobernanza (Echegoyemberry, 2017) y un disparador de políticas multiplicadoras a favor de las mujeres.

Hemos de aclarar que, evidentemente, aún quedan muchos interrogantes por resolver y realidades que documentar, en esta reflexión solo hemos bosquejado un trazado introductorio a estas temáticas que se espera que la academia, políticos y sociedad civil profundicen más en ella.

\section{Bibliografía}

BOURDIEU, P. (2010). Ia dominacion masculina. Obtenido de Editorial Anagrama: https://www.ocac.cl/wp-content/uploads/2015/01/Pierre-Bourdeu-Ladominaci\%C3\%B3n-masculina.pdf

BID (2016). 3 ventajas de la participación de las mujeres en la planificación urbana. Obtenido de: https://blogs.iadb.org/ciudades-sostenibles/es/mujeres/

CEDAW. (1997). Recomendación general 23, adoptada por el Comité para la Eliminación de la Discriminación contra la Mujer, $16^{\circ}$ período de sesiones, 1997, U.N. Doc. A/52/38.Obtenido de: http://hrlibrary.umn.edu/gencomm/Sgeneral23.htm

CEPAL. (2007). el aporte de las mujeres a la igualdad en America Latina y el Caribe. Obtenido de https://repositorio.cepal.org/bitstream/handle/11362/2855/1/ S3282007_es.pdf

CEPAL. (2014). Notas de Poblaciòn. Obtenido de: https://repositorio.cepal.org/ bitstream/handle/11362/37632/np99011037_es.pdf?sequence=1 
DAROS, William Roberto. (2014). La mujer posmoderna y el machismo. Franciscanum 162, Vol. Lvi. pg 107-129. Disponible en: http://www.scielo.org.co/pdf/frcn/ v56n162/v56n162a05.pdf

ECHEGOYEMBERRY, N. (2017). Identidad, género y Derechos Humanos: El rol de las mujeres en el cuidado de "la casa común" (ambiente). Revista Uruguaya de Antropología y Etnografía, 2(1), 55-70. Recuperado en 15 de junio de 2019, de: $\quad$ http://www.scielo.edu.uy/scielo.php?script=sci_arttext\&pid=S239368862017000100055\#B2

GARCIA NERT, Marta. (24 de junio de 1996). La calle como camino o tropiezo para la igualdad. Obtenido de http://habitat.aq.upm.es/boletin/n7/amgar.html

GONZALEZ, Jose. Maria. (1998). La cuestion urbana: Algunas perspectivas criticas. En C. d. constitucionales, Revista de estudios políticos (págs. 303-333). ESPAÑA: Centro de Estudios politicos y constitucionales. Disponible en: https://dialnet.unirioja.es/ servlet/articulo?codigo $=27496$

HAYDEN, Dolores (1980) ¿Cómo será una ciudad no sexista? especulaciones sobre vivienda, diseño urbano y empleo, en el curso: planificando y diseñando una sociedad no sexista. Los Ángeles: Universidad de California. Documento obtenido de https://vdocuments.site/dolores-hayden-what-would-a-non-sexist-city-be-like. html

IBÁÑEZ María luisa (2006). La participación de las mujeres en la planificación de las ciudades. Universidad de Salamanca. Disponible en:https://exaequo.apemestudos.org/files/2017-10/artigo-10-m.-luisa-martinez.pdf

JUSTO MORENO, aurora (1999) la planificación urbana desde la perspectiva de género. Por un derecho a la ciudad igualitaria. http://www.nodo50.org/ mujeresurbanistas/cursos/AJusto.html

LANZAROTE, Aida Guillen. (2011). El derecho a la ciudad, un derecho humano emergente. En Institut de Drets Humans de Catalunya. d. Catalunya, capitulo 1. El derecho a la ciudad de la Serie Derechos Humanos Emergentes. Número 7 (págs. 16-27). Barcelona, España : Disponible en: https://www.uclg-cisdp.org/sites/ default/files/DHE_7_esp_1.pdf

LIPOVETSKY, G. (2007). La tercera mujer, permanencia y revolucion de lo femenino. Barcelona: Anagrama (6a edicion). Disponible en: https://sinismos.files.wordpress. com/2013/02/109856484-gilles-lipovetsky-la-tercera-mujer.pdf

LIPOVETSKY, G. (2007). La tercera mujer, permanencia y revolucion de lo femenino. Barcelona: Anagrama (6a edicion). Disponible en: https://sinismos.files.wordpress. com/2013/02/109856484-gilles-lipovetsky-la-tercera-mujer.pdf 
MONJESILVA, A. (10 de mayo de 2016).¿Cómo mejorar la movilidad de las mujeres? Obtenido de https://blogs.iadb.org/transporte/es/como-mejorar-la-movilidad-delas-mujeres/

MUXI, Zaida. (2011). Reflexiones en torno a las mujeres y el derecho a la ciudad desde una realidad con espejismos. En Institut de Drets Humans de Catalunya. d. Catalunya, capitulo 1. El derecho a la ciudad de la Serie Derechos Humanos Emergentes. Número 7 (págs. 16-27). Barcelona, España : Disponible en: https:// www.uclg-cisdp.org/sites/default/files/DHE_7_esp_1.pdf

ONU MUJERES. (27 de febrero de 2019). Pregúntale a una senadora: ¿Cómo es que Bolivia tiene tan alta representación de mujeres en la política, y por qué es importante incluir las voces de las mujeres en la política? Obtenido de http://www.unwomen. org/es/news/stories/2019/2/ask-a-senator-about-politics-in-bolivia

ONU, MUJERES. (12 de marzo de 2019). De cinco ministros uno es mujer, según el nuevo mapa de la UIP-ONU Mujeres. Obtenido de http://www.unwomen.org/es/ news/stories/2019/3/press-release--ipu-un-women-map-women-in-politics

RANABOLDO, Claudia \& Solan Yolanda. (2008). "Desigualdad de género en la participación política de las mujeres en América Latina y el Caribe". RIMISP, Centro Latinoamericano para el Desarrollo Rural. Santiago, Chile.

RAINER, L., \& Marisol, D. (2011). Una ciudad al alcance de las mujeres, herramientas para incorporar el genero en el ordenamiento territorial, el caso de Bogota. Obtenido de Secretaria de Planeacion: https://undiaunaarquitecta.files.wordpress. com/2015/07/una-ciudad-al-alcance-de-las-mujeres.pdf

RAINERO, Liliana e Rodigou Maite (2003) Indicadores urbanos de género. Instrumentos para la gobernabilidad urbana 51 congreso internacional de americanistas. Santiago de chile disponible en http://www.redmujer.org.ar/ articulos.htm

TELLO, F., \& Silvia, V. (2009). La participación política de las mujeres en los gobiernos locales latinoamericanos: barreras y desafios para una efectiva democracia de genero. Obtenido de http://americalatinagenera.org/newsite/images/cdr-documents/ publicaciones/participacion_mujeres_gob_locales_flavia_mabel_tello.pdf

THE GUARDIAN. (05 de diciembre de 2014). If women built cities what would our urban landscape look like. Obtenido de https://www.theguardian.com/cities/2014/ dec/05/if-women-built-cities-what-would-our-urban-landscape-look-like

WORLD BANK. (5 de febrero de 2014). Gender, Travel and Job access: evidence from Buenos Aires. Obtenido de http://siteresources.worldbank.org/ INTURBANTRANSPORT/Resources/2014-Feb-5-Gender-and-Mobility.pdf 